\title{
Geometry of Orlicz spaces equipped with norms generated by some lattice norms in $\mathbb{R}^{2}$
}

\author{
Yunan Cui ${ }^{1} \cdot$ Henryk Hudzik ${ }^{2,3} \cdot$ Haifeng $\mathrm{Ma}^{4}$ \\ Received: 23 May 2018 / Accepted: 22 January 2019 / Published online: 5 March 2019 \\ (c) The Author(s) 2019
}

\begin{abstract}
In Orlicz spaces generated by convex Orlicz functions a family of norms generated by some lattice norms in $\mathbb{R}^{2}$ are defined and studied. This family of norms includes the family of the p-Amemiya norms $(1 \leq p \leq \infty)$ studied in Cui et al. (Nonlinear Anal. 69:1796-1816, 2008; Nonlinear Anal. 71:6343-6364, 2009; J. Math. Anal. Appl. 432:1095-1105, 2015; Nonlinear Anal. 75:3973-3993, 2012) and He et al. (Fixed Point Theory Appl. 2013:1-18, 2013). Criteria for strict monotonicity, lower and upper local uniform monotonicities and uniform monotonicities of Orlicz spaces and their subspaces of order continuous elements, equipped with these norms, are given in terms of the generating Orlicz functions, and the lattice norms in $\mathbb{R}^{2}$. The problems of strict convexity and of the existence of order almost isometric as well as order isometric copies in these spaces are also discussed.
\end{abstract}

Keywords Orlicz spaces $\cdot$ Norms generated by lattice norms in $\mathbb{R}^{2}$. Copies of $l^{\infty}$. Monotonicity properies $\cdot$ Strict convexity

Mathematics Subject Classification 46E30 - 46B20 - 46B45 - 46B42 - 46A80

Y. Cui: supported by NSF of China 11871181.

$凶$ Henryk Hudzik

hudzik@amu.edu.pl

Yunan Cui

cuiya@hrbust.edu.cn

Haifeng Ma

haifengma@aliyun.com

1 Department of Mathematics, Harbin University of Science and Technology, Harbin 150080, China

2 Faculty of Economics and Information Technology, The State University of Applied Sciences in Płock, ul., Gałczynskiego 28, 09-402 Płock, Poland

3 Faculty of Mathematics and Computer Science, Adam Mickiewicz University in Poznań, Umultowska 87, 61-614 Poznań, Poland

4 School of Mathematical Science, Harbin Normal University, Harbin 150025, China 


\section{Introduction}

Let $p(\cdot)$ be a lattice norm in $\mathbb{R}^{2}$ such that $p((1,0))=1$ and extended to $(0, \infty)$ and $(1, \infty)$ by $p((0, \infty))=p((1, \infty))=\infty$, if the point $(1, \infty)$ will be used. Let $\Phi: \mathbb{R} \rightarrow \mathbb{R}_{+}:=$ $[0,+\infty)$ be an Orlicz function, that is, $\Phi$ is a nonzero function vanishing at zero, $\Phi$ is convex and even. Let us define $a(\Phi):=\sup \{u \geq 0: \Phi(u)=0\}$.

In the following $(\Omega, \Sigma, \mu)$ is a $\sigma$-finite complete measure space and $L^{0}=L^{0}(\Omega, \Sigma, \mu)$ is the space of all (equivalence classes of) $\Sigma$-measurable functions $x: \Omega \rightarrow \mathbb{R}$, where functions $x$ and $y$ equal $\mu$-a.e. in $\Omega$ belong to the same class of equivalence (we simply say that they are identified).

Given any Orlicz function $\Phi$ we define on the space $L^{0}(\Omega, \Sigma, \mu)$ the functional

$$
I_{\Phi}(x)=\int_{\Omega} \Phi(x(t)) d \mu .
$$

It is easy to see that the functional $I_{\Phi}$ has the following properties:

(a) $I_{\Phi}: L^{0}(\Omega, \Sigma, \mu) \rightarrow \mathbb{R}_{+}^{e}:=\mathbb{R}_{+} \cup\{+\infty\}$,

(b) $I_{\Phi}$ is convex,

(c) $I_{\Phi}$ is even,

(d) $I_{\Phi}(0)=0$ and if $x \in L^{0}(\Omega, \Sigma, \mu) \backslash\{0\}$, then $I_{\Phi}(\lambda x) \neq 0$ for some $\lambda>0$,

and it is called a convex modular (see [38]). We are interested in introducing a norm generated by the functional $I_{\Phi}$ in the biggest possible subspace of $L^{0}(\Omega, \Sigma, \mu)$. This subspace is called the Orlicz space, denoted by $L^{\Phi}=L^{\Phi}(\Omega, \Sigma, \mu)$ and defined by (see $\left.[5,30,31,34,36,38,40]\right)$

$$
L^{\Phi}(\Omega, \Sigma, \mu):=\left\{x \in L^{0}(\Omega, \Sigma, \mu): I_{\Phi}(\lambda x)<\infty \text { for some } \lambda \in(0,+\infty)\right\} .
$$

Let us denote by $A_{\Phi}(1)$ the modular unit ball, that is,

$$
A_{\Phi}(1)=\left\{x \in L^{0}(\Omega, \Sigma, \mu): I_{\Phi}(x) \leq 1\right\} .
$$

Since the Orlicz function $\Phi$ is absolutely convex, that is,

$$
\Phi(\alpha u+\beta v) \leq|\alpha| \Phi(u)+|\beta| \Phi(v)
$$

for all $\mathrm{u}, v \in \mathbb{R}$ and all $\alpha, \beta \in \mathbb{R}$ with $|\alpha|+|\beta| \leq 1$, we obtain absolute convexity of the functional $I_{\Phi}$ and, in consequence, also absolute convexity of the set $A_{\Phi}(1)$. The Minkovski functional generated by the set $A_{\Phi}(1)$ has finite values for these elements from $L^{0}(\Omega, \Sigma, \mu)$ which are absorbing by $A_{\Phi}(1)$. It is easy to see that the biggest subspace of $L^{0}(\Omega, \Sigma, \mu)$, the elements of which are absorbed by $A_{\Phi}(1)$, that is

$$
\exists \lambda>0: \frac{x}{\lambda} \in A_{\Phi}(1)
$$

is just the Orlicz space $L^{\Phi}(\mu)$. The Minkovski functional of the set $A_{\Phi}(1)$ is called in the literature the Luxemburg norm, it is denoted by $\|\cdot\|_{\Phi}$ and defined by the formula (see $[5,35,36,38])$

$$
\|x\|_{\Phi}=\inf \left\{\lambda>0: I_{\Phi}\left(\frac{x}{\lambda}\right) \leq 1\right\}\left(\forall x \in L^{\Phi}(\mu)\right) .
$$

The following family of norms, called the p-Amemiya norms, was already defined and studied in Orlicz spaces for $0 \leq p \leq \infty$ :

$$
\|x\|_{\Phi, p}=\inf _{k>0} \frac{1}{k}\left(1+\left(I_{\Phi}(k x)\right)^{p}\right)^{\frac{1}{p}}\left(\forall x \in L^{\Phi}(\mu)\right),
$$


where $1 \leq p \leq \infty$ (see $[10,11,14,15,20])$. The norm $\|\cdot\|_{\Phi, 1}$ is the Orlicz norm $\|\cdot\|_{\Phi}^{0}$ which was defined by Orlicz in [39] by the formula

$$
\|x\|_{\Phi}^{0}=\sup \left\{\left|\int_{\Omega} x(t) y(t) d \mu\right|: y \in L^{0}(\Omega, \Sigma, \mu) \text { and } I_{\Phi^{*}}(y) \leq 1\right\},
$$

where $\Phi^{*}$ is the function complementary to $\Phi$ in the sense of Young, that is,

$$
\Phi^{*}(u)=\sup _{\nu \geq 0}\{|u| v-\Phi(v)\}
$$

For $p=\infty$, we have (see [27])

$$
\|x\|_{\Phi, \infty}=\lim _{p \rightarrow \infty} \frac{1}{k}\left(1+\left(I_{\Phi}(k x)\right)^{p}\right)^{\frac{1}{p}}=\inf _{k>0} \frac{1}{k} \max \left(1,\left(I_{\Phi}(k x)\right)\right)=\|x\|_{\Phi} .
$$

It is well known that all norms from the family $\left\{\|\cdot\|_{\Phi, p}\right\}_{p \in[1, \infty]}$ are equivalent and that the Luxemburg norm $\|\cdot\|_{\Phi}=\|x\|_{\Phi, \infty}$ is the smallest norm and the Orlicz norm $\|\cdot\|_{\Phi}^{0}=\|x\|_{\Phi, 1}$ is the biggest one. The Orlicz space $L^{\Phi}(\mu)$ equipped with every norm from this family of norms is a Banach space, which is even the Banach function lattice, called also the Köthe space (see $[2,3,30,31,34,40])$, which means that for any $p \in[1,+\infty]$, the space $\left(L^{\Phi}(\mu)\right.$, $\left.\|\cdot\|_{\Phi, p}\right)$ has the following properties:

$1^{\circ}$ For any $x \in L^{\circ}(\mu), y \in L^{\Phi}(\mu)$, if $|x(t)| \leq|y(t)|$ for $\mu$ a.e. $t \in \Omega$, then $x \in L^{\Phi}$ and $\|x\|_{\Phi, p} \leq\|y\|_{\Phi, p}$,

$2^{\circ}$ There exists a function $x \in L^{\Phi}(\mu)$ such that $x(t)>0$ for $\mu$-a.e. $t \in \Omega$.

The same properties has the space $\left(E^{\Phi}(\mu),\|\cdot\|_{\Phi, p}\right)$ defined below. Let us recall that an element $x$ of a Köthe space $\left(E,\|\cdot\|_{E}\right)$ is said to be order continuous if for any sequence $\left\{x_{n}\right\}_{n=1}^{\infty}$ in $E$ such that $0 \leq x_{n}(t) \leq|x(t)|$ for all $n \in \mathbb{N}$ and $\mu$-a.e. $t \in \Omega$, the condition $x_{n}(t) \rightarrow 0$ as $n \rightarrow \infty$ for $\mu$-a.e. $t \in \Omega$ implies that $\left\|x_{n}\right\|_{E} \rightarrow 0$ as $n \rightarrow \infty$. The set of all order continuous elements in $E$ is denoted by $E_{a}$, and the space $\left(E_{a},\|\cdot\|_{E}\right)$ is again a Kőthe space. It is obvious that equivalent norms keep the order continuity property. It is well known that (see [34,41])

$$
\left(L^{\Phi}(\mu)\right)_{a}=E^{\Phi}(\mu)
$$

where

$$
E^{\Phi}(\mu):=\left\{x \in L^{0}(\Omega, \Sigma, \mu): I_{\Phi}(\lambda x)<\infty \text { for any } \lambda>0\right\} .
$$

In this paper we will introduce a new family of norms in the Orlicz space $L^{\Phi}(\mu)$. Namely, given any lattice norm $p(\cdot)$ in $\mathbb{R}^{2}$ such that $p((1,0))=1$, which is assumed in the whole paper, we define the following functional in $L^{\Phi}(\mu)$ :

$$
\|x\|_{\Phi, p(\cdot)}:=\inf _{k>0} \frac{1}{k} p\left(\left(1, I_{\Phi}(k x)\right)\right)\left(\forall x \in L^{\Phi}(\mu)\right) .
$$

The set of all $k>0$ such that $\frac{1}{k} p\left(\left(1, I_{\Phi}(k x)\right)\right)=\|x\|_{\Phi, p(\cdot)}$ is denoted by $K(x)$. We will prove that such functionals are norms in $L^{\Phi}(\mu)$. Of course, these norms are lattice norms and they are equivalent each others. We will work on criteria for strict convexity and various their monotonicity properties (strict monotonicity, lower and upper local uniform monotonicity and uniform monotonicity) as well as on order almost isometric copies of $l^{\infty}$ and order isometric copies of $l^{\infty}$. 
We need to define all others notions that will be used in this paper. A Banach lattice $X=(X, \leq,\|\cdot\|)$, for the definition of which we refer to [2,30,34,41], is said to be strictly monotone if for any $x, y \in X$ such that $0 \leq x \leq y$ and $x \neq y$, we have $\|x\|<\|y\|$. By the homogenity of the norm $\|\cdot\|$, we can restrict ourselves in this definition to $y \geq 0$ satisfying $\|y\|=1$. Let us denote by $X_{+}$the positive cone in $X$, that is, the set of all $x \in X$ such that $x \geq 0$. In our definitions below $X$ always denotes a Banach lattice $(X, \leq,\|\cdot\|)$. $X$ is said to be uniformly monotone (see [2,32]) if for any $\varepsilon \in(0,1)$ there exists $\delta(\varepsilon) \in(0,1)$ such that if $x, y \in X, 0 \leq x \leq y$; $\|x\| \geq \varepsilon$ and $\|y\|=1$, then $\|y-x\| \leq 1-\delta(\varepsilon)$. The biggest function $\delta_{X}:(0,1) \rightarrow(0,1)$ with this property, that is, the function

$$
\delta_{X}(\varepsilon)=\inf \{1-\|y-x\|: 0 \leq x \leq y ;\|x\| \geq \varepsilon,\|y\|=1\},
$$

is called the modulus of monotonicity of $X$ (see [2] and for the properties of $\delta_{X}(\cdot)$ also [23]). It is known (see [32]) that $X$ is uniformly monotone if and only if for any $\varepsilon>0$ there exists $\sigma(\varepsilon)>0$ such that for any $x, y \in X_{+}$such that $\|x\| \geq \varepsilon$ and $\|y\|=1$ there holds $\|y+x\| \geq 1+\sigma(\varepsilon)$. $X$ is said to be lower (upper) locally uniformly monotone if for any $y \in X_{+}$with $\|y\|=1$ and any $\varepsilon \in(0,1)$ (resp. any $\varepsilon>0$ ) there exists $\delta(y, \varepsilon) \in(0,1)$ (resp. $\sigma(y, \varepsilon)>0$ ) such that for any $x \in X$ satisfying $0 \leq x \leq y$ and $\|x\| \geq \varepsilon$ (resp. $x \geq 0$ with $\|x\| \geq \varepsilon$ ), we have $\|y-x\| \leq 1-\delta(y, \varepsilon)$ (resp. $\|y+x\| \geq 1+\sigma(y, \varepsilon)$ ). For the definition of these two properties see $[2,4,24]$.

It is obvious that $\Phi$ vanishes only at 0 iff $a(\Phi)=0$. For any Orlicz function $\Phi$ we say that it satisfies condition $\Delta_{2}\left(\mathbb{R}_{+}\right)\left(\Phi \in \Delta_{2}\left(\mathbb{R}_{+}\right)\right.$for short) if there exists $K>0$ such that $\Phi(2 u) \leq K \Phi(u)$ for any $u \geq 0$. We say that $\Phi$ satisfies condition $\Delta_{2}$ at infinity $\left(\Phi \in \Delta_{2}(\infty)\right.$ for short) if there are positive constants $u_{0}$ and $K$ such that $\Phi(2 u) \leq K \Phi(u)$ for all $u \geq u_{0}$. We say that $\Phi$ satisfies condition $\Delta_{2}$ at zero $\left(\Phi \in \Delta_{2}(0)\right.$ for short) if there exist two positive constants $u_{0}$ and $K$ such that $\Phi\left(u_{0}\right)>0$ and $\Phi(2 u) \leq K \Phi(u)$ for all $u \in\left[0, u_{0}\right]$. It is easy to see that $\Delta_{2}\left(\mathbb{R}_{+}\right)$if and only if $\Phi \in \Delta_{2}(\infty)$ and $\Phi \in \Delta_{2}(0)$.

The $\Delta_{2}$-condition for $\Phi$ should be defined suitably to the measure space $(\Omega, \Sigma, \mu)$ in such a way that the corresponding Orlicz space $\left(L^{\Phi}(\mu),\|\cdot\|_{\Phi}\right)$ is order continuous. We know that suitable $\Delta_{2}$-condition for the couple $(\Phi,(\Omega, \Sigma, \mu))$ is the following:

(a) condition $\Delta_{2}\left(\mathbb{R}_{+}\right)$if $(\Omega, \Sigma, \mu)$ is infinite and non-atomic.

(b) condition $\Delta_{2}(\infty)$ if $(\Omega, \Sigma, \mu)$ is finite and non-atomic.

(c) condition $\Delta_{2}(0)$ if $\Omega=\mathbb{N}, \Sigma=2^{\mathbb{N}}$ and $\mu$ is the counting measure on $2^{\mathbb{N}}$.

It is obvious that any $\sigma$-finite measure space $(\Omega, \Sigma, \mu)$ can be represented as the direct sum of two measure spaces $\left(\Omega_{n-a}, \Sigma \cap \Omega_{n-a},\left.\mu\right|_{\Sigma \cap \Omega_{n-a}}\right) \oplus\left(\Omega_{a}, \Sigma \cap \Omega_{a},\left.\mu\right|_{\Sigma \cap \Omega_{a}}\right)$, where $\Omega_{a}$ is the set of all atoms for $\mu$ in $\Sigma$ and $\Omega_{n-a}=\Omega \backslash \Omega_{a}$.

If $\Omega_{a}$ is finite and $\mu\left(\Omega_{n-a}\right)>0$, then the suitable condition $\Delta_{2}$ for $\Phi$ is the $\Delta_{2}$-condition for the non-atomic measure space defined above. If $\mu\left(\Omega_{n-a}\right)=0$ and $\Omega_{a}=\mathbb{N}, \Sigma=2^{\mathbb{N}}$ and $\mu$ is the counting measure on $2^{\mathbb{N}}$, then the suitable condition $\Delta_{2}$ is the condition $\Delta_{2}(0)$. If $\mu\left(\Omega_{n-a}\right)>0, \Omega_{a}=\mathbb{N}, \Sigma=2^{\mathbb{N}}$ and $\mu$ is the counting measure on $2^{\mathbb{N}}$, then the suitable $\Delta_{2-}$ condition for $\Phi$ is the conjunction of the suitable $\Delta_{2}$-condition for the non-atomic measure space and of the condition $\Delta_{2}(0)$, that is, condition $\Delta_{2}\left(R_{+}\right)$. In our paper, we always assume that all atoms have the measure 1 and we identify the atoms with the singletons $\{n\}$, where $n \in \mathbb{N}$ (the set of all natural numbers).

Monotonicity properties of Banach lattices have applications in the dominated best approximation (see $[6,8,14,17,24,32]$ ) and in the fixed point theory (see $[14,17,20])$. They are also strongly related to the complex rotundity properties (see [29]). For these reasons monotonicity properties were investigated in various classes of function spaces. Namely, in 
[13,24,26,32,33] for Musielak-Orlicz spaces, in [21] for Lorentz spaces, in [19] for OrliczLorentz spaces, in $[4,14,16,18,23,37]$ for Orlicz spaces, in [28,29] for Calderòn-Lozanovskiı̌ spaces, in [12] for Cesàro-Orlicz sequence spaces, for Orlicz-Sobolev spaces in [8]. Relationships between monotonicity properties and rofundity properties as well as between monotonicity properties and orthogonal monotonicity properties in Köthe spaces were given in [22]. In abstract Banach lattices relationships between monotonicity properties and dominated best approximation problems were studied in $[6,14,17]$.

Problems on estimates or calculations of the characteristic of monotonicity in Orlicz spaces and Orlicz-Lorentz spaces were studied in $[16,18,19,23]$. Applications of the monotonicity properties in the ergodic theory in Banach lattices were studied in [1].

Theorem 1 For any lattice norm $p(\cdot)$ on $\mathbb{R}^{2}$ such that $p((1,0))=p((0,1))=1$ we have the inequality

$$
\forall(u, v) \in \mathbb{R}^{2}: \quad \max (|u|,|v|) \leq p((u, v)) \leq|u|+|v|,
$$

that is, the smallest (resp.the biggest) lattice norm $p(\cdot)$ among these ones with $p((1,0))=$ $p((0,1))=1$ is the $l^{\infty}$-norm (resp. $l^{1}$-norm). Moreover,

$$
\max (|1|,|v|) \leq p((1, v)) \leq|1|+|v|
$$

for any $v \in \mathbb{R}$ and the norm $\|\cdot\|_{\Phi, p(\cdot)}$ is non-smaller than the Luxemburg norm and nongreater than the Orlicz norm for any Orlicz function $\Phi$ and any lattice norm $p(\cdot)$ on $\mathbb{R}^{2}$.

Proof Let us take any $(u, v) \in \mathbb{R}^{2}$. Then

$$
\begin{aligned}
p((u, v)) & =p((|u|,|v|)) \geq p((|u|, 0))=p(|u|(1,0)) \\
& =|u| p((1,0))=|u|
\end{aligned}
$$

and

$$
\begin{aligned}
p((u, v)) & =p((|u|,|v|)) \geq p((0,|v|))=: p(|v|(0,1)) \\
& =|v| p((0,1))=|v|,
\end{aligned}
$$

whence

$$
p((u, v)) \geq \max (|u|,|v|)=p_{\infty}((u, v)) .
$$

On the other hand for any $(u, v) \in \mathbb{R}^{2}$, we have

$$
\begin{aligned}
p((u, v)) & =p((|u|,|v|))=p((|u|, 0)+(0,|v|)) \\
& \leq p((|u|, 0))+p((0,|v|))=|u| p((1,0))+|v| p((0,1)) \\
& =|u|+|v|=: p_{1}((u, v)) .
\end{aligned}
$$

The second part of the theorem follows by the inequalities from the first sentence of this theorem and from the facts that $\|\cdot\|_{\Phi, p_{\infty}(\cdot)}$ is equal to the Luxemburg norm and $\|\cdot\|_{\Phi, p_{1}(\cdot)}$ is equal to the Orlicz norm as well as to the Amemiya norm (see [27]).

Geometry of Orlicz spaces equipped with the $p$-Amemiya norm, that is, the norm $\|\cdot\|_{p(\cdot)}$, where $p((u, v)):=\left(|u|^{p}+|v|^{p}\right)^{\frac{1}{p}}$ for any $(u, v) \in \mathbb{R}^{2}$, was considered in the papers $[10,11,14,15]$, the fixed point property in these spaces was studied in $[14,20]$. The dominated best approximation in these spaces was studied in [14].

Theorem 2 For any Orlicz function $\Phi$ and any lattice norm $p(\cdot)$ in $\mathbb{R}^{2}$ the functional $\|\cdot\|_{\Phi, p(\cdot)}$ is a norm in $L^{\Phi}(\mu)$. 
Proof We have $I_{\Phi}(k 0)=0$ for any $k>0$, so

$$
\|0\|_{\Phi, p(\cdot)}=\inf _{k>0} \frac{1}{k} p((1,0))=\inf _{k>0} \frac{1}{k}=0 .
$$

Let us assume that $x \in L^{\Phi}(\mu) \backslash\{0\}$. Since $\Phi$ is a nonzero function, that is, there exists $u_{0}>0$ such that $\Phi\left(u_{0}\right)>0$, so there exists $k_{0}>0$ such that $I_{\Phi}\left(k_{0} x\right)>1$. Then

$$
\begin{aligned}
\|x\|_{\Phi, p(\cdot)} & =\min \left(\inf _{0<k \leq k_{0}} \frac{1}{k} p\left(\left(1, I_{\Phi}(k x)\right)\right), \inf _{k \geq k_{0}} \frac{1}{k} p\left(\left(1, I_{\Phi}(k x)\right)\right)\right) \\
& \geq \min \left(\inf _{0<k \leq k_{0}} \frac{1}{k} p((1,0)), \inf _{k \geq k_{0}} \frac{1}{k} p\left(\left(0, I_{\Phi}(k x)\right)\right)\right) \\
& \geq \min \left(\frac{1}{k_{0}}, \inf _{k \geq k_{0}} p\left(\left(0, \frac{1}{k} I_{\Phi}(k x)\right)\right)\right) \\
& =\min \left(\frac{1}{k_{0}}, p\left(\left(0, \frac{1}{k_{0}} I_{\Phi}\left(k_{0} x\right)\right)\right)\right) \\
& >0 .
\end{aligned}
$$

Now, we will show that the functional $\|\cdot\|_{\Phi, p(\cdot)}$ is absolutely homogeneous. Let us take any $x \in L^{\Phi}(\mu)$ and any $\lambda>0$. If $x=0$, then $\lambda x=0$ for any $\lambda \in \mathbb{R}$, whence

$$
\|\lambda 0\|_{\Phi, p(\cdot)}=\|0\|_{\Phi, p(\cdot)}=0=\lambda \cdot 0=\lambda\|0\|_{\Phi, p(\cdot)} .
$$

So let us assume that $x \neq 0$. Then

$$
\begin{aligned}
\|\lambda x\|_{\Phi, p(\cdot)} & =\inf _{k>0} \frac{1}{k} p\left(\left(1, I_{\Phi}(k|\lambda| x)\right)\right) \\
& =\inf _{k>0} \frac{|\lambda|}{|\lambda| k} p\left(\left(1, I_{\Phi}(k \lambda x)\right)\right) \\
& =|\lambda| \inf _{k>0} \frac{1}{|\lambda| k} p\left(\left(1, I_{\Phi}(k|\lambda| x)\right)\right) \\
& =|\lambda|\|x\|_{\Phi, p(\cdot) .}
\end{aligned}
$$

Finally, we will show that the functional $\|\cdot\|_{\Phi, p(\cdot)}$ satisfies the triangle inequality. Let us take arbitrary $x, y \in L^{\Phi}(\mu)$. If at least one element among $x$ and $y$ is equal to zero function, then the triangle inequality is obvious. So assume that $x \neq 0$ and $y \neq 0$. Let us take any $\varepsilon>0$. There exists constants $\lambda>0$ and $l>0$ such that

$$
\begin{aligned}
\frac{1}{\lambda} p\left(\left(1, I_{\Phi}(\lambda x)\right)\right) & \leq\|x\|_{\Phi, p(\cdot)}+\varepsilon, \\
\frac{1}{l} p\left(\left(1, I_{\Phi}(l y)\right)\right) & \leq\|y\|_{\Phi, p(\cdot)}+\varepsilon .
\end{aligned}
$$

Then

$$
\begin{aligned}
\|x+y\|_{\Phi, p(\cdot)} & \leq \frac{\lambda+l}{\lambda l} p\left(\left(1, I_{\Phi}\left(\frac{\lambda l}{\lambda+l}(x+y)\right)\right)\right) \\
& =\frac{\lambda+l}{\lambda l} p\left(\left(1, I_{\Phi}\left(\frac{l}{\lambda+l}(\lambda x)+\frac{\lambda}{\lambda+l}(l y)\right)\right)\right) \\
& =\frac{\lambda+l}{\lambda l} p\left(\left(\frac{l}{\lambda+l}+\frac{\lambda}{\lambda+l}, I_{\Phi}\left(\frac{l}{\lambda+l}(\lambda x)+\frac{\lambda}{\lambda+l}(l y)\right)\right)\right)
\end{aligned}
$$




$$
\begin{aligned}
& \leq \frac{\lambda+l}{\lambda l} p\left(\left(\frac{l}{\lambda+l}+\frac{\lambda}{\lambda+l}, \frac{l}{\lambda+l} I_{\Phi}(\lambda x)+\frac{\lambda}{\lambda+l} I_{\Phi}(l y)\right)\right) \\
& =\frac{\lambda+l}{\lambda l} p\left(\left(\frac{l}{\lambda+l}, \frac{l}{\lambda+l} I_{\Phi}(\lambda x)\right)+\left(\frac{\lambda}{\lambda+l}, \frac{\lambda}{\lambda+l} I_{\Phi}(l y)\right)\right) \\
& \leq \frac{\lambda+l}{\lambda l}\left\{\frac{l}{\lambda+l} p\left(\left(1, I_{\Phi}(\lambda x)\right)\right)+\frac{\lambda}{\lambda+l} p\left(\left(1, I_{\Phi}(l y)\right)\right)\right\} \\
& =\frac{1}{\lambda} p\left(\left(1, I_{\Phi}(\lambda x)\right)\right)+\frac{1}{l} p\left(\left(1, I_{\Phi}(l y)\right)\right) \\
& \leq\|x\|_{\Phi, p(\cdot)}+\|y\|_{\Phi, p(\cdot)}+2 \varepsilon .
\end{aligned}
$$

By the arbitrariness of $\varepsilon>0$, we obtain the inequality

$$
\|x+y\|_{\Phi, p(\cdot)} \leq\|x\|_{\Phi, p(\cdot)}+\|y\|_{\Phi, p(\cdot)},
$$

which finishes the proof of the theorem.

Lemma 1 Let $p(\cdot)$ be a lattice norm in $\mathbb{R}^{2}$ extended to $(0, \infty)$ and $(1, \infty)$ by $p((0, \infty))=$ $p((1, \infty))=\infty$. Then: $(i)$ if $\Phi$ is an Orlicz function satisfying the condition $\lim _{u \rightarrow+\infty}$ $(\Phi(u) / u)=+\infty$, we have that for any $x \in L^{\Phi}(\mu) \backslash\{0\}$ there exists $l \in(0,+\infty)$ such that

$$
\|x\|_{\Phi, p(\cdot)}=\frac{1}{l} p\left(\left(1, I_{\Phi}(l x)\right)\right) .
$$

(ii) For any Orlicz function $\Phi$, if $x \in L^{\Phi}(\mu)$ and $K(x)=\emptyset$, then

$$
\|x\|_{\Phi, p(\cdot)}=A p((0,1))\|x\|_{L^{1}(\Omega)},
$$

where $A=\lim _{u \rightarrow \infty}(\Phi(u) / u)$.

Proof $(i)$ Let $x \in L^{\Phi}(\mu) \backslash\{0\}$. The condition $(\Phi(u) / u) \rightarrow+\infty$ as $u \rightarrow+\infty$ implies that

$$
\frac{1}{k} I_{\Phi}(k x) \rightarrow+\infty \text { as } k \rightarrow+\infty
$$

whence we get

$$
\text { (1) } \begin{aligned}
\lim _{k \rightarrow+\infty} \frac{1}{k} p\left(\left(1, I_{\Phi}(k x)\right)\right) & \geq \lim _{k \rightarrow+\infty} \frac{1}{k} p\left(\left(0, I_{\Phi}(k x)\right)\right) \\
& =\lim _{k \rightarrow+\infty} \frac{1}{k} I_{\Phi}(k x) p((0,1))=+\infty
\end{aligned}
$$

Moreover,

$$
\lim _{k \rightarrow 0^{+}} \frac{1}{k} p\left(\left(1, I_{\Phi}(k x)\right)\right) \geq \lim _{k \rightarrow 0^{+}} \frac{1}{k} p((1,0))=\lim _{k \rightarrow 0^{+}} \frac{1}{k}=+\infty,
$$

Hence and from condition (1), we get that there exist positive constants $k_{0}$ and $k_{1}$ such that $k_{0}<k_{1}<+\infty, I_{\Phi}\left(k_{1} x\right)<\infty$, and

$$
\|x\|_{\Phi, p(\cdot)}=\inf _{k_{0} \leq k \leq k_{1}} \frac{1}{k} p\left(\left(1, I_{\Phi}(k x)\right)\right) .
$$

The function $\left.f(k):=I_{\Phi}(k x)\right)$ is convex and it has finite values on the compact interval $\left[k_{0}, k_{1}\right]$, so it is continuous on this interval. In consequence, by continuity of the norm $p(\cdot)$, the function $g:\left[k_{0}, k_{1}\right] \rightarrow \mathbb{R}_{+}$defined by

$$
g(k)=p\left(\left(\frac{1}{k}, \frac{1}{k} I_{\Phi}(k x)\right)\right)
$$


is also continuous on the interval $\left[k_{0}, k_{1}\right]$. Therefore, the desired number $l \in(0,+\infty)$ exists.

(ii) Let $x \in L^{\Phi}(\mu)$ and $K(x)=\phi$. We can assume without loss of generality that $x \neq 0$. It is obvious that the infimum in the definition of the norm $\|x\|_{\Phi, p(\cdot)}$ can be restricted to such $k>0$ that $I_{\Phi}(k x)<\infty$. Since, $\|x\|_{\Phi, p(\cdot)}<\infty$ and $\lim _{k \rightarrow 0_{+}} \frac{1}{k} p\left(\left(1, I_{\Phi}(\alpha x)\right)\right)=\infty$, there exists $k_{0}>0$ such that

$$
\|x\|_{\Phi, p(\cdot)}=\inf _{k \in\left[k_{0}, \infty\right)} \frac{1}{k} p\left(\left(1, I_{\Phi}(\alpha x)\right)\right)
$$

Since for any $k_{1} \in\left[k_{0}, \infty\right)$ such that $I_{\Phi}\left(k_{1} x\right)<\infty$, by continuity of the function $f(k) \doteq$ $I_{\Phi}(k x)$ on the interval $\left[k_{0}, k_{1}\right]$, there exists $l \in\left[k_{0}, k_{1}\right]$ such that

$$
\inf _{k \in\left[k_{0}, k_{1}\right]} \frac{1}{k} p\left(\left(1, I_{\Phi}(\alpha x)\right)\right)=\frac{1}{l} p\left(\left(1, I_{\Phi}(l x)\right)\right)
$$

and $K(x)=\phi$, we conclude by continuity of the norm $p(\cdot)$ that

$$
\begin{aligned}
\|x\|_{\Phi, p(\cdot)} & =\lim _{k \rightarrow \infty} \frac{1}{k} p\left(\left(1, I_{\Phi}(\alpha x)\right)\right) \\
& =\lim _{k \rightarrow \infty} p\left(\left(\frac{1}{k}, \frac{1}{k} I_{\Phi}(\alpha x)\right)\right) \\
& =p\left(\left(\lim _{k \rightarrow \infty} \frac{1}{k}, \lim _{k \rightarrow \infty} \frac{1}{k} I_{\Phi}(\alpha x)\right)\right) \\
& =p\left(\left(0, \lim _{k \rightarrow \infty} \frac{1}{k} I_{\Phi}(\alpha x)\right)\right) .
\end{aligned}
$$

Let us note that, by the Beppo Levi theorem,

$$
\begin{aligned}
\lim _{k \rightarrow \infty} \frac{1}{k} I_{\Phi}(k x) & =\lim _{k \rightarrow \infty} \frac{1}{k} \int_{\Omega} \Phi(k x(t)) d \mu \\
& =\lim _{k \rightarrow \infty} \int_{\text {supp } x} \frac{\Phi(k x(t))}{k|x(t)|}|x(t)| d \mu \\
& =\int_{\text {supp } x}\left(\lim _{k \rightarrow \infty} \frac{\Phi(k x(t))}{k|x(t)|}\right)|x(t)| d \mu \\
& =A \int_{\Omega}|x(t)| d \mu \\
& =A\|x\|_{L^{1}(\Omega)} .
\end{aligned}
$$

whence, by the previous equalitices, we get

$$
\|x\|_{\Phi, p(\cdot)}=A p((0,1))\|x\|_{L^{1}(\Omega)} .
$$

Lemma 2 Let $\Phi$ be any Orlicz function and $p$ be any lattice norm on $\mathbb{R}^{2}$ such that $p((1,0))=$ $p((0,1))=1$ and extended to $(1, \infty)$ by $p((1, \infty))=\infty$. Then

$$
\|x\|_{\Phi, p(\cdot)} \leq 1+I_{\Phi}(x)
$$

for any $x \in L^{\Phi}(\mu)$. Moreover, if $I_{\Phi}(\lambda x)=\infty$ for any $\lambda>1$, then

$$
1 \leq\|x\|_{\Phi, p(\cdot)} .
$$


Proof By Theorem 1, we have

$$
\|x\|_{\Phi, p(\cdot)}=\inf _{k>0} \frac{1}{k} p\left(\left(1, I_{\Phi}(k x)\right)\right) \leq p\left(\left(1, I_{\Phi}(x)\right)\right) \leq 1+I_{\Phi}(x) .
$$

Simultaneously, if $I_{\Phi}(\lambda x)=\infty$ for any $\lambda>1$, then

$$
\begin{aligned}
\|x\|_{\Phi, p(\cdot)} & =\inf _{k>0} \frac{1}{k} p\left(\left(1, I_{\Phi}(k x)\right)\right) \\
& =\min \left(\inf _{0<k \leq 1} \frac{1}{k} p\left(\left(1, I_{\Phi}(k x)\right)\right), \inf _{k \geq 1} \frac{1}{k} p\left(\left(1, I_{\Phi}(k x)\right)\right)\right) \\
& \geq \min \left(\inf _{0<k \leq 1} \frac{1}{k} p((1,0)), p\left(\left(1, I_{\Phi}(x)\right)\right)\right) \\
& =\min \left(1, p\left(\left(1, I_{\Phi}(x)\right)\right)\right)
\end{aligned}
$$

Theorem 3 Let $p(\cdot)$ be $\overline{\bar{e}}$ a norm on $\mathbb{R}^{2}$ as in Lemma 2. If $\Phi$ is an Orlicz function $\Phi$ which does not satisfy suitable $\Delta_{2}$-condition, then $\left(L^{\Phi}(\mu),\|\cdot\|_{\Phi, p(\cdot)}\right)$ contains an order linearly almost isometric copy of $l^{\infty}$, that is, for any $\varepsilon>0$ there exists a linear nonnegative operator $P_{\varepsilon}: l^{\infty} \rightarrow L^{\Phi}(\mu)$ such that

$$
\|z\|_{\infty} \leq\|P z\|_{\Phi, p(\cdot)} \leq(1+\varepsilon)\|z\|_{\infty} \quad\left(\forall z \in l^{\infty}\right) .
$$

Proof Under the assumptions on $\Phi$, given any $\varepsilon>0$, there exists a sequence $\left\{x_{n}\right\}_{n=1}^{\infty}$ in $L^{\Phi}(\mu)$ with pairwise disjoint supports and such that $I_{\Phi}\left(x_{n}\right) \leq \varepsilon / 2^{n}, x_{n} \geq 0$ and $I_{\Phi}\left(\lambda x_{n}\right)=$ $\infty$ for any $n \in \mathbb{N}$ and $\lambda>1$ (see [5]). Let us define an operator $P_{\varepsilon}$ on $l^{\infty}$ by the formula

$$
P_{\varepsilon} z=\sum_{n=1}^{\infty} z_{n} x_{n} \quad\left(\forall z=\left\{z_{n}\right\}_{n=1} \in l^{\infty}\right),
$$

where the series is defined on $\Omega$ pointwisely. It is obvious that $P_{\varepsilon}$ is linear and nonnegative. By pairwise disjointness of the supports of the element $x_{n} \in L^{\Phi}(\mu)$, there is no problem with the pointwise convergence of the series, because for any $t \in \Omega$ there exists at least one $n \in N$ such that $t \in \operatorname{suppx}_{n}$. It is easy to see that $P_{\varepsilon} z \in L^{\Phi}(\mu)$ for any $z \in l^{\infty}$. Indeed,

$$
I_{\Phi}\left(\frac{P_{\varepsilon} z}{\|z\|_{\infty}}\right)=\sum_{n=1}^{\infty} I_{\Phi}\left(\frac{z_{n}}{\|z\|_{\infty}} x_{n}\right) \leq \sum_{n=1}^{\infty} \frac{z_{n}}{\|z\|_{\infty}} I_{\Phi}\left(x_{n}\right) \leq \sum_{n=1}^{\infty} \frac{\varepsilon}{2^{n}}=\varepsilon .
$$

Let us note first that for any $z \in l^{\infty}$ and $k>0$, we have

$$
\begin{aligned}
p\left(\left(1, I_{\Phi}\left(k \frac{P_{\varepsilon} z}{\|z\|_{\infty}}\right)\right)\right) & =p\left(\left(1, I_{\Phi}\left(k \frac{P_{\varepsilon}|z|}{\|z\|_{\infty}}\right)\right)\right) \\
& =p\left(\left(1, I_{\Phi}\left(k \frac{\sum_{n=1}^{\infty}\left|z_{n}\right| x_{n}}{\|z\|_{\infty}}\right)\right)\right) \leq p\left(\left(1, I_{\Phi}\left(k \sum_{n=1}^{\infty} x_{n}\right)\right)\right) \\
& =p\left(\left(1, \sum_{n=1}^{\infty} I_{\Phi}\left(k x_{n}\right)\right)\right) .
\end{aligned}
$$

In consequence, by Lemma 2, we have for any $z=\left\{z_{n}\right\}_{n=1}^{\infty} \in l^{\infty}$, 


$$
\begin{aligned}
\| \frac{P_{\varepsilon} z}{\|z\|_{\infty} \|_{\Phi, p(\cdot)}} & =\inf _{k>0} \frac{1}{k} p\left(\left(1, I_{\Phi}\left(k \frac{P_{\varepsilon} z}{\|z\|_{\infty}}\right)\right)\right) \\
& \leq p\left(\left(1, I_{\Phi}\left(\frac{P_{\varepsilon} z}{\|z\|_{\infty}}\right)\right)\right) \\
& \leq p\left(\left(1, I_{\Phi}\left(\sum_{n=1}^{\infty} x_{n}\right)\right)\right) \\
& \leq 1+\varepsilon,
\end{aligned}
$$

whence $\left\|P_{\varepsilon} z\right\|_{\Phi, p(\cdot)} \leq(1+\varepsilon)\|z\|_{\infty}$ for any $z \in l^{\infty}$.

On the other hand, since for any $\lambda>0$ there exist $n_{\lambda} \in N$ such that $\lambda\left|z_{n_{\lambda}}\right| /\|z\|_{\infty}>1$, we have

$$
I_{\Phi}\left(\frac{P_{\varepsilon} \lambda z}{\|z\|_{\infty}}\right) \geq I_{\Phi}\left(\frac{\lambda\left|z_{n_{\lambda}}\right|}{\|z\|_{\infty}} x_{n_{\lambda}}\right)=\infty,
$$

whence, by Lemma 2,

$$
\left\|\frac{P_{\varepsilon} \lambda z}{\|z\|_{\infty}}\right\|_{\Phi, p(\cdot)}=\left\|\frac{\lambda P_{\varepsilon} z}{\|z\|_{\infty}}\right\|_{\Phi, p(\cdot)} \geq 1,
$$

that is,

$$
\left\|P_{\varepsilon} z\right\|_{\Phi, p(\cdot)} \geq \frac{\|z\|_{\infty}}{\lambda}
$$

By the arbitrariness of $\lambda>1$, we have $\left\|P_{\varepsilon} z\right\|_{\Phi, p(\cdot)} \geq\|z\|_{\infty}$ for any $z \in l^{\infty}$, which finishes the proof.

Theorem 4 Let $p(\cdot)$ be a norm in $\mathbb{R}^{2}$ such as in Lemma 2 and $\Phi$ be an Orlicz function with $a(\Phi):=\sup \{u \geq 0: \Phi(u)\}>0$. Then in both cases, a non-atomic infinite measure space as well as the case of the counting measure on $2^{N}$, the Orlicz space $\left(L^{\Phi}(\mu),\|\cdot\|_{\Phi, p(\cdot)}\right)$ contains a linearly order isometric copy of $l^{\infty}$.

Proof Under the assumptions on the measure space, there exists a sequence $\left\{A_{n}\right\}_{n=1}^{\infty}$ of pairwise disjoint sets with $\mu\left(A_{n}\right)=+\infty$ for any $n \in N$. Let us define

$$
x_{n}=a(\Phi) \mathcal{X}_{A_{n}}(\forall n \in N), x:=\sum_{n=1}^{\infty} x_{n}=\sup _{n \in N} x_{n},
$$

where the series is defined pointwisely (no problem with its pointwise convergence because of pairwise disjointness of the sets $\left.A_{n}\right)$. It is obvious that $I_{\Phi}(x)=0$ and $I_{\Phi}\left(x_{n}\right)=0$ as well as that $I_{\Phi}(\lambda x)=I_{\Phi}\left(\lambda x_{n}\right)=+\infty$ for any $n \in N$ and $\lambda>1$. Moreover, for any $n \in \mathbb{N}$,

$$
\begin{aligned}
\left\|x_{n}\right\|_{\Phi, p(\cdot)} & =\min _{0<k \leq 1}\left(\inf _{0<1} \frac{1}{k} p\left(\left(1, I_{\Phi}\left(k x_{n}\right)\right)\right), \inf _{k \geq 1} \frac{1}{k} p\left(\left(1, I_{\Phi}\left(k x_{n}\right)\right)\right)\right) \\
& =\inf _{0<k \leq 1} \frac{1}{k} p\left(\left(1, I_{\Phi}\left(k x_{n}\right)\right)\right) \\
& =\inf _{0<k \leq 1} \frac{1}{k} p((1,0))=p((1,0))=1 .
\end{aligned}
$$


In the same way, we can prove that $\|x\|_{\Phi, p(\cdot)}=1$. Let us define the following operator on $l^{\infty}$ :

$$
P z=\sum_{n=1}^{\infty} z_{n} x_{n} \quad\left(\forall z=\left\{z_{n}\right\} \in l^{\infty}\right)
$$

Let us first note that $P: l^{\infty} \longrightarrow L^{\Phi}(\mu)$. Namely, $I_{\Phi}\left(\frac{P z}{\|z\|_{\infty}}\right) \leq I_{\Phi}\left(\sum_{n=1}^{\infty} x_{n}\right)=I_{\Phi}(x)=0$, whence $P z \in L^{\Phi}(\mu)$ for any $z \in l^{\infty}$. Moreover,

$$
\begin{aligned}
\left\|\frac{P z}{\|z\|_{\infty}}\right\|_{\Phi, p(\cdot)} & =\inf _{k>0} \frac{1}{k} p\left(\left(1, I_{\Phi}\left(k \frac{P z}{\|z\|_{\infty}}\right)\right)\right) \\
& \leq \inf _{k>0} \frac{1}{k} p\left(\left(1, I_{\Phi}(k x)\right)\right) \\
& =\|x\|_{\Phi, p(\cdot)} \\
& =1
\end{aligned}
$$

whence $\|P z\|_{\Phi, p(\cdot)} \leq\|z\|_{\infty}$. On the other hand, given any $\lambda>1$, one can find $n_{\lambda} \in N$ such that $\lambda\left|z_{n_{\lambda}}\right|>\|z\|_{\infty}$. Consequently,

$$
\left\|\frac{\lambda P z}{\|z\|_{\infty}}\right\|_{\Phi, p(\cdot)}=\left\|\frac{P(\lambda z)}{\|z\|_{\infty}}\right\|_{\Phi, p(\cdot)} \geq\left\|x_{n_{\lambda}}\right\|_{\Phi, p(\cdot)}=1,
$$

whence $\|P z\|_{\Phi, p(\cdot)} \geq \frac{\|z\|_{\infty}}{\lambda}$. By the arbitrariness of $\lambda>1$, we obtain that $\|P z\|_{\Phi, p(\cdot)} \geq$ $\|z\|_{\infty}$, which together with the opposite inequality (proved already) gives the equality $\|P z\|_{\Phi, p(\cdot)}=\|z\|_{\infty}$ for any $z \in l^{\infty}$, which means that $P$ is an isometry. It is obvious that the operator $P$ is linear. Since the functions $x_{n}$ are non-negative, so $P$ is also non-negative, that is, $P z \geq 0$ for any $z \in l^{\infty}, z \geq 0$. In consequence, the operator $P$ is a linear order isometry, which finishes the proof.

Theorem 5 Let $p(\cdot)$ be a lattice norm in $\mathbb{R}^{2}$ which is strictly increasing on the vertical halfline $\left\{(1, u): u \in R_{+}\right\}$in $\mathbb{R}^{2}$ and let $\Phi$ be a strictly convex Orlicz function. Let the couple $(\Phi, p(\cdot))$ satisfy the condition

(2) $K(x) \neq \emptyset \forall x \in L^{\Phi}(\mu) \backslash\{0\}$

Then the Orlicz space $\left(L^{\Phi}(\mu),\|\cdot\|_{\Phi, p(\cdot)}\right)$ is strictly convex.

Proof Assume that $x, y \in S\left(\left(L_{+}^{\Phi}(\mu),\|\cdot\|_{\Phi, p(\cdot)}\right)\right)$ and $x \neq y$. Then $\Phi \circ k x \neq \Phi \circ k y$ for any $k \in(0,+\infty)$ because strict convexity of $\Phi$ implies that $\Phi$ is a $1-1$ function on $R_{+}$. We also have that $\Phi \circ k \frac{x+y}{2} \leq \frac{1}{2}\{\Phi \circ k x+\Phi \circ k y\}$ and $\Phi \circ k \frac{x+y}{2} \neq \frac{1}{2}\{\Phi \circ k x+\Phi \circ k y\}$ for any $k \in(0,+\infty)$. Let $\lambda, l \in(0,+\infty)$ be such that

$$
\begin{aligned}
\|x\|_{\Phi, p(\cdot)} & =\frac{1}{\lambda} p\left(\left(1, I_{\Phi}(\lambda x)\right)\right), \\
\|y\|_{\Phi, p(\cdot)} & =\frac{1}{l} p\left(\left(1, I_{\Phi}(l y)\right)\right),
\end{aligned}
$$

and define $q=\frac{2 \lambda l}{\lambda+l}$. Let us note that $\lambda x \neq l y$. Indeed, assuming that $\lambda x=l y$, we get by $\|x\|_{\Phi, p(\cdot)}=\|y\|_{\Phi, p(\cdot)}$ that $\lambda=l$ whence, by $x=y$, a contradiction. Then by strict convexity of $I_{\Phi}$ and the fact that $p(\cdot)$ is strictly increasing on the half-line $\left\{(1, u): u \in R_{+}\right\} \subset R^{2}$, we get 


$$
\begin{aligned}
\left\|\frac{x+y}{2}\right\|_{\Phi, p(\cdot)} & \leq \frac{1}{q} p\left(\left(1, I_{\Phi}\left(q \frac{x+y}{2}\right)\right)\right) \\
& =\frac{1}{q} p\left(\left(1, I_{\Phi}\left(\frac{l}{l+\lambda} \lambda x+\frac{\lambda}{l+\lambda} l y\right)\right)\right) \\
& <\frac{1}{q} p\left(\left(1, \frac{l}{l+\lambda} I_{\Phi}(\lambda x)+\frac{\lambda}{l+\lambda} I_{\Phi}(l y)\right)\right) \\
& =\frac{1}{q} p\left(\left(\frac{l}{l+\lambda}+\frac{\lambda}{l+\lambda}, \frac{l}{l+\lambda} I_{\Phi}(\lambda x)+\frac{\lambda}{l+\lambda} I_{\Phi}(l y)\right)\right) \\
& =\frac{1}{q} p\left(\frac{l}{l+\lambda}\left(1, I_{\Phi}(\lambda x)+\frac{\lambda}{l+\lambda}\left(1, I_{\Phi}(l y)\right)\right)\right) \\
& \leq \frac{1}{q}\left\{\frac{l}{l+\lambda} p\left(\left(1, I_{\Phi}(\lambda x)\right)\right)+\frac{\lambda}{l+\lambda} p\left(\left(1, I_{\Phi}(l y)\right)\right)\right\} \\
& =\frac{1}{2}\left\{\frac{1}{\lambda} p\left(\left(1, I_{\Phi}(\lambda x)\right)\right)+\frac{1}{l} p\left(\left(1, I_{\Phi}(l y)\right)\right)\right\} \\
& =\frac{1}{2}\left\{\|x\|_{\Phi, p(\cdot)}+\|y\|_{\Phi, p(\cdot)}\right\} \\
& =\frac{1}{2}\{1+1\} \\
& =1
\end{aligned}
$$

which finishes the proof that the positive cone $\left(L_{+}^{\Phi}(\mu),\|\cdot\|_{\Phi, p(\cdot)}\right)$ is strictly convex. But then we obtain from a general result from [22] that the whole space $\left(L_{\Phi}(\mu),\|\cdot\|_{\Phi, p(\cdot)}\right)$ is also strictly convex.

Corollary 1 [5] Under the assumption that $\Phi$ is a strictly convex Orlicz function such that $\sup _{u>0}[A u-\Phi(u)]=\infty$, where $A:=\lim _{u \rightarrow \infty}\left(\frac{\Phi(u)}{u}\right)$, which gives that $K(x) \neq 0$ for any $x \in L^{\Phi}(\mu) \backslash\{0\}$, the Orlicz space $L^{\Phi}(\mu)$ equipped with the Orlicz norm is strictly convex.

Proof Under the assumptions on $\Phi$, condition (2) from Theorem 5 is satisfied (see [7]). Moreover, the Orlicz norm in $L^{\Phi}(\mu)$ is just the norm $\|\cdot\|_{\Phi, p(\cdot)}$ with $p((u, v))=|u|+|v|$ for any $(u, v) \in \mathbb{R}^{2}$. Since this norm $p(\cdot)$ is strictly increasing on the vertical half-line $\left\{(1, u): u \in \mathbb{R}_{+}\right\}$in $R^{2}$, the thesis of our corollary follows directly form Theorem 5 .

Theorem 6 Assume that $p(\cdot)$ is a lattice norm in $\mathbb{R}^{2}$ which is strictly increasing on the vertical half-line $\left\{(1, u): u \in \mathbb{R}_{+}\right\} \subseteq \mathbb{R}^{2}$ and $\Phi$ is an Orlicz function such that $K(x) \neq \varnothing$ for any $x \in L^{\Phi}(\mu) \backslash\{0\}$. Then the following statements are equivalent:

(i) $a(\Phi)=0$,

(ii) $\left(L^{\Phi}(\mu),\|\cdot\|_{\Phi, p(\cdot)}\right)$ is strictly monotone,

(iii) $\left(E^{\Phi}(\mu),\|\cdot\|_{\Phi, p(\cdot)}\right)$ is strictly monotone.

Proof $(i) \Longrightarrow($ ii $)$. Let $0 \leq x \leq y \in S\left(L^{\Phi}(\mu),\|\cdot\|_{\Phi, p(\cdot)}\right)$ and $x \neq y$. We know, by the assumption, that

$$
\|y\|_{\Phi, p(\cdot)}=\frac{1}{l} p\left(\left(1, I_{\Phi}(l y)\right)\right)
$$

for some $l \in(0,+\infty)$. Since $0 \leq l x \leq l y$ and $l x \neq l y$, and by the assumption that $a(\Phi)=0$ the Orlicz function $\Phi$ is strictly increasing on $\mathbb{R}_{+}$, we obtain

$$
I_{\Phi}(l x)<I_{\Phi}(l y) .
$$


In consequence

$$
\begin{aligned}
\|x\|_{\Phi, p(\cdot)} & \leq \frac{1}{l} p\left(\left(1, I_{\Phi}(l x)\right)\right)<\frac{1}{l} p\left(\left(1, I_{\Phi}(l y)\right)\right) \\
& =\|y\|_{\Phi, p(\cdot),}
\end{aligned}
$$

which finishes the proof of the implication $(i) \Longrightarrow($ ii $)$. The implication $(i i) \Longrightarrow($ iii $)$ is obvious.

(iii) $\Longrightarrow($ i $)$. Assuming that $(i)$ does not hold, we will prove that ( $i$ ii $)$ does not hold. Take any $y \in E^{\Phi}(\mu)$ such that $y \geq 0,\|y\|_{\Phi, p(\cdot)}=1$ and the set $A:=\Omega \backslash$ suppy has positive measure. Define $z=y+\frac{a(\Phi)}{k} \mathcal{X}_{A}$, where $k \in K(y)$. Then $z \in E^{\Phi}(\mu)$ and $\|z\|_{\Phi, p(\cdot)} \geq 1$ because $|z(t)| \geq|y(t)|$ for $\mu-$ a.e. $t \in \Omega$. On the other hand $\|z\|_{\Phi, p(\cdot)} \leq \frac{1}{k} p\left(\left(1, I_{\Phi}(k z)\right)\right)=$ $\frac{1}{k} p\left(\left(1, I_{\Phi}(k y)\right)\right)=\|y\|_{\Phi, p(\cdot)}=1$. Therefore, $\|z\|_{\Phi, p(\cdot)}=1$, whence $(i i i)$ does not hold.

Remark 1 Let us note that the assumption that the norm $p(\cdot)$ is strictly increasing on the half-line $\left\{(1, u): u \in \mathbb{R}_{+}\right\}$is not in general necessary neither for strict convexity nor for strict monotonicity of the space $\left(L^{\Phi}(\mu),\|\cdot\|_{\Phi, p(\cdot)}\right)$. Namely, if $p((u, v))=\max (|u|, \mid$ $u \mid)\left(\forall(u, v) \in \mathbb{R}^{2}\right)$, then $p(\cdot)$ is not strictly increasing on the half-line $\left\{(1, u): u \in \mathbb{R}_{+}\right\}$, but if $\Phi$ satisfies suitable $\Delta_{2}$-condition, then the space $\left(L^{\Phi}(\mu),\|\cdot\|_{\Phi, p(\cdot)}\right)$ is strictly convex whenever $\Phi$ is strictly convex, and $\left(L^{\Phi}(\mu),\|\cdot\|_{\Phi, p(\cdot)}\right)$ is strictly monotone, whenever $a(\Phi)=0$, see [24,32] (because $\|\cdot\|_{\Phi, p(\cdot)}$ is then equal to the Luxemburg norm).

Corollary 2 It follows from Theorem 4 that if $\Phi$ is an Orlicz function with $a(\Phi)>0$ and if the measure space is non-atomic and infinite or the counting measure on $2^{N}$, then under the assumptions of Theorem 4 on the norm $p(\cdot)$, the space $\left(L^{\Phi}(\mu),\|\cdot\|_{\Phi, p(\cdot)}\right)$ is not strictly monotone, because $\left(l^{\infty},\|\cdot\|_{\infty}\right)$ is not strictly monotone (namely, the elements $x=(1,0,0, \cdots)$ and $y=(1,1,0,0, \cdots)$ satisfy the conditions $0 \leq x \leq y, x \neq y$, $\left.\|x\|_{\infty}=\|y\|_{\infty}=1\right)$. Therefore, $\left(L^{\Phi}(\mu),\|\cdot\|_{\Phi, p(\cdot)}\right)$ has no monotonicity property nonweaker than strict monotonicity. It is also not strictly convex (because any strictly convex Banach lattice is strictly monotone), so it has no convexity property non-weaker than strict convexity.

Theorem 7 Assume that $p(\cdot)$ is a lattice norm on $\mathbb{R}^{2}$ which is strictly monotone and $p((1,0))=p((0,1))=1$. Let $\Phi$ be an Orlicz function; $x, y \in L^{\Phi}(\mu)$ and $0 \leq x \leq$ $y \in S\left(\left(L^{\Phi}(\mu),\|\cdot\|_{\Phi, p(\cdot))}\right)\right.$.

Then

(3) $\|y-x\|_{\Phi, p(\cdot)} \leq 1-\delta_{m, p(\cdot)}\left(I_{\Phi}(x)\right)$, wherever $I_{\phi}(x)<1$ or $\delta_{m, p(\cdot)}$ is left continuous at the point 1

where $\delta_{m, p(\cdot)}(\cdot)$ is the modulus of monotonicity of the space $\left(\mathbb{R}^{2}, p(\cdot)\right)$.

Proof First let us note that since $\mathbb{R}^{2}$ is finitely dimensional, by strict monotonicity under the norm $p(\cdot)$, the space $\left(\mathbb{R}^{2}, p(\cdot)\right)$ is uniformly monotone. Therefore, $\delta_{m, p(\cdot)}(\varepsilon)>0$ for any $\varepsilon \in(0,1)$. For the definition of the modulus of monotonicity and its properties see [23].

Let us take $x$ and $y$ mentioned in the theorem and any $\varepsilon>0$. By the definition of the norm $\|\cdot\|_{\Phi, p(\cdot)}$, there exists $l \in(0,+\infty)$ such that

$$
\frac{1}{l} p\left(\left(1, I_{\Phi}(l y)\right)\right) \leq\|y\|_{\Phi, p(\cdot)}+\varepsilon=1+\varepsilon .
$$

Since $p\left(\left(1, I_{\Phi}(l y)\right)\right) \geq p((1,0))=1$, the previous inequality implies that $\frac{1}{l} \leq 1+\varepsilon$, whence $l \geq \frac{1}{1+\varepsilon}$. Hence, by the assumption that $p((0,1))=1$, we have 
(4) $p\left(\left(0, \frac{1}{l} I_{\Phi}(l x)\right)\right)=\frac{1}{l} I_{\Phi}(l x) p((0,1)) \geq(1+\varepsilon) I_{\Phi}\left(\frac{x}{1+\varepsilon}\right)$.

By convexity of the modular $I_{\Phi(\cdot)}$, we obtain

$$
0 \leq(1+\varepsilon) I_{\Phi}\left(\frac{x}{1+\varepsilon}\right) \leq I_{\Phi}(x) \leq\|x\|_{\Phi, p(\cdot)} \leq\|y\|_{\Phi, p(\cdot)}=1 .
$$

By superadditivity of $\Phi$ on $\mathbb{R}_{+}$, we have superadditivity of the modular $I_{\Phi}$ on $\left(L^{\Phi}(\mu)\right)_{+}$. Hence, and by the equality

$$
p\left(\left(0, \frac{1}{l} I_{\Phi}(l x)\right)\right)=\frac{1}{l} I_{\Phi}(l x) p((0,1))=\frac{1}{l} I_{\Phi}(l x),
$$

we obtain

$$
\begin{aligned}
\|y-x\|_{\Phi, p(\cdot)} & \leq \frac{1}{l} p\left(\left(1, I_{\Phi}(l(y-x))\right)\right) \\
& \leq \frac{1}{l} p\left(\left(1, I_{\Phi}(l y)-I_{\Phi}(l x)\right)\right) \\
& =p\left(\left(\frac{1}{l}, \frac{1}{l} I_{\Phi}(l y)\right)-\left(0, \frac{1}{l} I_{\Phi}(l x)\right)\right) \\
& \leq \frac{1}{l} p\left(\left(1, I_{\Phi}(l y)\right)\right)-\delta_{m, p(\cdot)}\left(p\left(\left(0, \frac{1}{l} I_{\Phi}(l x)\right)\right)\right) \\
& \leq 1+\varepsilon-\delta_{m, p(\cdot)}\left((1+\varepsilon) I_{\Phi}\left(\frac{x}{1+\varepsilon}\right)\right) .
\end{aligned}
$$

Taking in place of $\varepsilon$ a sequence $\left\{\varepsilon_{n}\right\}_{n=1}^{\infty}$ such that $\varepsilon_{n} \searrow 0$ as $n \nearrow \infty$ and applying the Beppo Levi theorem, we obtain that $\left(1+\varepsilon_{n}\right) I_{\Phi}\left(\frac{x}{1+\varepsilon_{n}}\right) \nearrow I_{\Phi}(x)$ as $n \nearrow \infty$. This means that $\lim _{\varepsilon \rightarrow 0^{+}}(1+\varepsilon) I_{\Phi}\left(\frac{x}{1+\varepsilon}\right) \rightarrow I_{\Phi}(x)$. Since the modulus of monotonicity is continuous on the interval $[0,1)$ and, by the assumption also at the point 1 if $I_{\phi}(\mathrm{x})=1$, we obtain the desired inequality.

Remark 2 If $p(\cdot)$ is a lattice norm in $\mathbb{R}^{2}$ as in Theorem 1 and $\Phi$ is an Orlicz function, then the Orlicz space $\left(L^{\Phi}(\mu),\|\cdot\|_{\Phi, p(\cdot)}\right)$ is order continuous if and only if $\Phi$ satisfies suitable $\Delta_{2}$-condition .

Proof Since all norms in $\mathbb{R}^{2}$ are equivalent, our norms $\|\cdot\|_{\Phi, p(\cdot)}$ are equivalent to the norm $\|\cdot\|_{\Phi, p_{\infty}(\cdot)}$, where $p_{\infty}((u, v))=\max (|u|,|v|)$. It is known (see $\left.[8,10]\right)$ that the norm $\|\cdot\|_{\Phi, p_{\infty}(\cdot)}$ is equal to the Luxemburg norm $\|\cdot\|_{\Phi}$. It is also well known (see [5,9]) that the Orlicz space $\left(L^{\Phi}(\mu),\|\cdot\|_{\Phi}\right)$ is order continuous if and only if $\Phi$ satisfies suitable $\Delta_{2}$ condition. Since order continuity is preserved by equivalent norms, we obtain that the space $\left(L^{\Phi}(\mu),\|\cdot\|_{\Phi, p(\cdot)}\right)$ is order continuous if and only if $\Phi$ satisfies suitable $\Delta_{2}$-condition.

Remark 3 It is known (see [5]) that for any sequence $\left\{x_{n}\right\}_{n=1}^{\infty}$ in $L^{\Phi}(\mu)$, with $I_{\Phi}\left(x_{n}\right) \rightarrow 0$ as $n \rightarrow \infty$, we have $\left\|x_{n}\right\|_{\Phi} \rightarrow 0$ as $n \rightarrow \infty$ if and only if $a(\Phi)=0$ and $\Phi$ satisfies the suitable $\Delta_{2}$-condition. Since for any lattice norm $p(\cdot)$ in $\mathbb{R}^{2}$ with $p((1,0))=1=p((0,1))$, the norm $\|\cdot\|_{\Phi, p(\cdot)}$ is equivalent to the Luxemburg norm $\|\cdot\|_{\Phi}$ in $L^{\Phi}(\mu)$, we have the same dependence between $I_{\Phi}\left(x_{n}\right) \rightarrow 0$ and $\left\|x_{n}\right\|_{\Phi, p(\cdot)} \rightarrow 0$ as $n \rightarrow \infty$. Namely, it holds if and only if $a(\Phi)=0$ and $\Phi$ satisfies suitable $\Delta_{2}$-condition. This dependence is equivalent to the fact that

$$
(\forall \varepsilon>0)(\exists \delta(\varepsilon)>0)\left(\forall x \in L^{\Phi}(\mu)\right)\left(\|x\|_{\Phi, p_{(\cdot)}} \geq \varepsilon \Rightarrow I_{\Phi}(x) \geq \delta(\varepsilon)\right) .
$$

Theorem 8 Let $p(\cdot)$ be a strictly monotone lattice norm in $\mathbb{R}^{2}$ such that $p((1,0))=$ $p((0,1))=1$ with the modulus of monotonicity left continuous at the point 1 and $\Phi$ be an Orlicz function. Then the assumption $a(\Phi)=0$ implies that: 
(i) $\left(L^{\Phi}(\mu),\|\cdot\|_{\Phi, p(\cdot)}\right)$ is strictly monotone,

(ii) $\left(E^{\Phi}(\mu),\|\cdot\|_{\Phi, p(\cdot)}\right)$ is lower locally uniformly monotone.

Assuming additionally that the couple $(\Phi, p(\cdot))$ is such that $K(x) \neq \varnothing$ for any $x \in$ $E^{\Phi}(\mu) \backslash\{0\}$ the conditions $a(\Phi)=0,(i),($ ii $)$ and

(iii) $\left(E^{\Phi}(\mu),\|\cdot\|_{\Phi, p(\cdot)}\right)$ is strictly monotone,

are equivalent.

Proof Under the assumption on $p(\cdot)$, we have by Theorem 7 that for any $x, y \in L^{\Phi}(\mu)$ such that $0 \leq x \leq y,\|y\|_{\Phi, p(\cdot)}=1$ and $\|x\|_{\Phi, p(\cdot)} \geq \varepsilon$, where $\varepsilon \in(0,1)$, we have

$$
\|y-x\|_{\Phi, p(\cdot)} \leq 1-\delta_{m, p(\cdot)}\left(I_{\Phi}(x)\right),
$$

where $\delta_{m, p(\cdot)}$ is the modulus of monotonicity of the space $\left(\mathbb{R}^{2}, p(\cdot)\right)$, which is uniformly monotone as a strictly monotone finite dimensional Banach space. Therefore, $\delta_{m, p(\cdot)}\left(I_{\Phi}(x)\right)>0$ by the fact that $a(\Phi)=0$ implies that $I_{\Phi}(x)>0$. In consequence $\|y-x\|_{\Phi, p(\cdot)}<1$, which means that property (i) holds.

Assume now that $x$ and $y$ are as above, but they belong to the space $E^{\Phi}(\mu)$. Let us note that the space $\left(E^{\Phi}(\mu),\|\|_{\Phi, p(\cdot)}\right)$ has the following property.

$$
\begin{aligned}
& \forall y \in S_{+}\left(\left(E^{\Phi}(\mu),\|\cdot\|_{\Phi, p(\cdot)}\right)\right), \quad \forall \varepsilon \in(0,1), \quad \exists \delta(y, \varepsilon)>0, \\
& \text { such that for any } 0 \leq x \leq y:\|x\|_{\Phi, p(\cdot)} \geq \varepsilon \Rightarrow I_{\Phi}(x) \geq \delta(y, \varepsilon) .
\end{aligned}
$$

Indeed, if this property does not hold, then there exist $y \in S_{+}\left(\left(L^{\Phi}(\mu),\|\cdot\|_{\Phi, p(\cdot)}\right)\right), \varepsilon \in(0,1)$ and a sequence $\left\{x_{n}\right\}_{n=1}^{\infty}$ in $E^{\Phi}(\mu)$ such that $\left\|x_{n}\right\|_{\Phi, p(\cdot)} \geq \varepsilon, 0 \leq x_{n} \leq y$ for any $n \in N$, and $I_{\Phi}\left(x_{n}\right) \rightarrow 0$ as $n \rightarrow \infty$. However, the last condition implies that $x_{n} \rightarrow 0$ in measure as $n \rightarrow \infty$. Hence, by the assumption that the measure space $(\Omega, \Sigma, \mu)$ is $\sigma$-finite, there exists a subsequence $\left\{x_{n_{k}}\right\}_{k=1}^{\infty}$ of $\left\{x_{n}\right\}_{n=1}^{\infty}$ such that $x_{n_{k}} \rightarrow 0$ as $k \rightarrow \infty \mu-$ a.e. in $\Omega$. Hence $\Phi \circ \lambda x_{n_{k}} \rightarrow 0 \mu-$ a.e. in $\Omega$ as $k \rightarrow \infty$ for any $\lambda>0$. Since, by the assumption that $y \in E^{\Phi}(\mu)$, we have $\Phi \circ \lambda y \in L^{1}(\mu)$ for any $\lambda>0$ and, by $0 \leq x_{n} \leq y$ for any $n \in N$, we have $\Phi \circ \lambda x_{n_{k}} \leq \Phi \circ \lambda y$ for any $k \in N$ and $\lambda>0$, the Lebesgue dominated convergence theorem implies that $I_{\Phi}\left(\lambda x_{n_{k}}\right)=\left\|\Phi \circ \lambda x_{n_{k}}\right\|_{L^{1}(\mu)} \rightarrow 0$ as $k \rightarrow \infty$ for any $\lambda>0$, which means that $\left\|x_{n_{k}}\right\|_{\Phi, p(\cdot)} \rightarrow 0$ as $k \rightarrow \infty$, a contradiction, which proves property (6) of $E^{\Phi}(\mu)$. Conditions (5) and (6) yield

$$
\|y-x\|_{\Phi, p(\cdot)} \leq 1-\delta_{m, p(\cdot)}(\delta(y, \varepsilon)),
$$

where $\delta(y, \varepsilon)$ does not depend on $x$, which means that property (ii) of $E^{\Phi}(\mu)$ holds.

It is obvious that (ii) $\Rightarrow$ (iii). In order to finish the proof, we need only to show that under the assumption that $K(x) \neq \emptyset$ for any $x \in E^{\Phi}(\mu) \backslash\{0\}$, condition (iii) implies that $a(\Phi)=0$. Assume that $a(\Phi)>0$ and $K(x) \neq \emptyset$ for any $x \in E^{\Phi}(\mu) \backslash\{0\}$. Let us take any $x \in E^{\Phi}(\mu)$ such that $x \geq 0,\|x\|_{\Phi, p(\cdot)}=1$ and $\mu(A)>0$, where $A=\Omega \backslash$ suppx. Defining $y=x+\frac{a(\Phi)}{k} \mathcal{X}_{A}$, where $k \in K(x)$, we have $0 \leq x \leq y \in E^{\Phi}(\mu)$ and $x \neq y$. Hence $\|y\|_{\Phi, p(\cdot)} \geq 1$. On the other hand, by $I_{\Phi}(k y)=I_{\Phi}(k x)$, we have

$$
\|y\|_{\Phi, p(\cdot)} \leq \frac{1}{k} p\left(\left(1, I_{\Phi}(k y)\right)\right)=\frac{1}{k} p\left(\left(1, I_{\Phi}(k x)\right)\right)=\|x\|_{\Phi, p(\cdot)}=1 .
$$

Therefore, property (iii) of $\left(E^{\Phi}(\mu),\|\cdot\|_{\Phi, p(\cdot)}\right)$ does not hold if $a(\Phi)>0$, and the proof of our theorem is complete.

Theorem 9 Let $p(\cdot)$ be a strictly monotone lattice norm on $\mathbb{R}^{2}$ as in Theorem 8 and $\Phi$ be an Orlicz function. Consider the following conditions: 
(i) $a(\Phi)=0$ and $\Phi$ satisfies suitable $\Delta_{2}$-condition,

(ii) $\left(L^{\Phi}(\mu),\|\cdot\|_{\Phi, p(\cdot)}\right)$ is uniformly monotone,

(iii) $\left(L^{\Phi}(\mu),\|\cdot\|_{\Phi, p(\cdot)}\right)$ is upper locally uniformly monotone,

(iv) $\left(E^{\Phi}(\mu),\|\cdot\|_{\Phi, p(\cdot)}\right)$ is upper locally uniformly monotone.

Then $(i) \Rightarrow($ ii $) \Rightarrow($ iii $) \Rightarrow($ iv $)$. Assuming additionally that $K(x) \neq \emptyset$ for any $x \in E^{\Phi}(\mu) \backslash\{0\}$, we have also that $(i v) \Rightarrow(i)$, whence we have then that all these four conditions are equivalent.

Proof (i) $\Rightarrow$ (ii). Assume that condition (i) is satisfied and that $0 \leq x \leq y \in S\left(\left(L^{\Phi}(\mu)\right.\right.$, $\left.\left.\|\cdot\|_{\Phi, p(\cdot)}\right)\right)$ and $\|x\|_{\Phi, p(\cdot)} \geq \varepsilon$. By the assumption that $\Phi$ satisfies suitable $\Delta_{2}-$ condition, we know that for any sequence $\left\{x_{n}\right\}_{n=1}^{\infty}$ in $L^{\Phi}(\mu)$, the conditions $I_{\Phi}\left(x_{n}\right) \rightarrow 0$ as $n \rightarrow \infty$ and $\left\|x_{n}\right\|_{\Phi, p(\cdot)} \rightarrow 0$ as $n \rightarrow \infty$ are equivalent. Therefore, there exists $\delta(\varepsilon) \in(0,1)$ such that $I_{\Phi}(x) \geq \delta(\varepsilon)$. By inequality (3) from Theorem 7, we have

$$
\|y-x\|_{\Phi, p(\cdot)} \leq 1-\delta_{m, p(\cdot)}\left(I_{\Phi}(x)\right) \leq 1-\delta_{m, p(\cdot)}(\delta(\varepsilon)) .
$$

Since $\delta_{m, p(\cdot)}(\delta(\varepsilon)) \in(0,1)$, property (ii) holds.

The implications (ii) $\Rightarrow$ (iii) $\Rightarrow$ (iv) are obvious, so in order to finish our proof, we need only to prove that (iv) $\Rightarrow$ (i), whenever $K(x) \neq \emptyset$ for any $x \in E(\mu) \backslash\{0\}$. Assume that condition (i) is not satisfied and $K(x) \neq \varnothing$ for any $x \in E^{\Phi}(\mu) \backslash\{0\}$. Then we have the alternative of the conditions: $a(\Phi)>0$ and $K(x) \neq 0$ for any $x \in E^{\Phi}(\mu) \backslash\{0\}$ or $\Phi \notin \Delta_{2}$ and $K(x) \neq \emptyset$ for any $x \in E^{\Phi}(\mu) \backslash\{0\}$.

In the first situation, by virtue of Theorem 6, condition (iv) does not hold, because $\left(E^{\Phi}(\mu),\|\cdot\|_{\Phi, p(\cdot)}\right)$ is not then strictly monotone.

In the second situation take a set $A \in \Sigma$ such that $0<\mu(A)<\mu(\Omega)$. By Proposition 2.1 in [9] there exists a sequence $\left\{y_{n}\right\}_{n=1}^{\infty}$ in $E^{\Phi}(\mu)$ such that $\operatorname{suppy}_{n} \subset T \backslash A, \frac{1}{1+2^{-n}} \leq$ $\left\|y_{n}\right\|_{\Phi}$ and $I_{\Phi}\left(y_{n}\right) \leq 2^{-n}$ for any $n \in N$. Then $\left\|y_{n}\right\|_{\Phi, p(\cdot)} \geq\left\|y_{n}\right\|_{\Phi} \geq \frac{1}{1+2^{-n}}$ for any $n \in N$. Take any $x \in E^{\Phi}(\mu)$ such that supp $x \subset A$ and $\|x\|_{\Phi, p(\cdot)}=1$. Let $k \in K(x)$. Then $k \geq 1$, because for any $k \in(0,1)$ we have

$$
\frac{1}{k} p\left(\left(1, I_{\Phi}(k x)\right)\right) \geq \frac{1}{k} p((1,0))=\frac{1}{k}>1=\|x\|_{\Phi, p(\cdot),}
$$

which means that $k \notin K(x)$. Defining $x_{n}=\frac{1}{k} y_{n}$, we have $\left\|x_{n}\right\|_{\Phi, p(\cdot)}=\left\|y_{n}\right\|_{\Phi, p(\cdot)} k \geq$ $\frac{1}{k\left(1+2^{-n}\right)} \geq \frac{2}{3 k}$. Moreover, $I_{\Phi}\left(k x_{n}\right)=I_{\Phi}\left(y_{n}\right) \leq 2^{-n}$, whence

$$
\begin{aligned}
\left\|x+x_{n}\right\|_{\Phi, p(\cdot)} & \left.\leq \frac{1}{k} p\left(\left(1, I_{\Phi}\left(k\left(x+x_{n}\right)\right)\right)\right)=\frac{1}{k} p\left(\left(1, I_{\Phi}(k x)\right)+I_{\Phi}\left(k x_{n}\right)\right)\right) \\
& =\frac{1}{k} p\left(\left(1, I_{\Phi}(k x)\right)+\left(0, I_{\Phi}\left(k x_{n}\right)\right)\right) \\
& \leq \frac{1}{k} p\left(\left(1, I_{\Phi}(k(x))\right)+\frac{1}{k} p\left(\left(0, I_{\Phi}\left(k\left(x_{n}\right)\right)\right)\right.\right. \\
& \leq\|x\|_{\Phi, p(\cdot)}+\frac{1}{k} p\left(\left(0,2^{-n}\right)\right) \\
& \leq 1+2^{-n}
\end{aligned}
$$

which together with $\left\|x_{n}\right\|_{\Phi, p(\cdot)} \geq \frac{2}{3 k}$ for any $n \in N$ means that the space $\left(E^{\Phi}(\mu),\|\cdot\|_{\Phi, p(\cdot)}\right)$ is not locally upper uniformly monotone. This finishes the proof. 
Acknowledgements Henryk Hudzik and Haifeng Ma gratefully acknowledge the support of the bilateral project between China and Poland (No. 37-18).

Open Access This article is distributed under the terms of the Creative Commons Attribution 4.0 International License (http://creativecommons.org/licenses/by/4.0/), which permits unrestricted use, distribution, and reproduction in any medium, provided you give appropriate credit to the original author(s) and the source, provide a link to the Creative Commons license, and indicate if changes were made.

\section{References}

1. Akcoglu, M.A., Sucheston, L.: On uniform monotonicity of norms and ergodic theorems in function spaces. Rend. Circ. Mat. Palermo (2) Suppl 8((2) Suppl), 325-335 (1985)

2. Birkhoff, G.: Lattice Theory. American Mathematical Society, Providence (1967)

3. Bru, B., Heinich, H.: Approximation dans les espaces de Kőthe. C. R. Math. Acad. Sci. Paris. 296, 773-775 (1983)

4. Bru, B., Heinich, H.: Monotonies des espaces de Orlicz (Monotonicities of Orlicz spaces), Comptes Rendus de la Academie des Sciences - Series I - Mathematics, (1985)

5. Chen, S.T.: Geometry of Orlicz Spaces. Diss. Math. 356, 1-221 (1996)

6. Chen, S.T., He, H., Hudzik, H.: Monotonicity and best approximation in Banach lattices. Acta. Math. Sin. (English Ser.) 25(5), 785-794 (2009)

7. Chen, S., Cui, Y, A., Hudzik, H.: Isometric copies of $l^{1}$ and $l^{\infty}$ in Orlicz spaces equipped with the Orlicz Norm. Proc. Amer. Math. Soc 132(2), 473-480 (2004)

8. Chen, S., He, X., Hudzik, H.: Monotonicity and best approximation in Orlicz-Sobolev spaces with the Luxemburg norm. Math. Anal. Appl. 344(2), 687-698 (2008)

9. Chen, S.T., Hudzik, H., Sun, H.Y.: Complemented copies of $l^{1}$ in Orlicz spaces. Math. Nachr. 158, 299-309 (1992)

10. Cui, Y.A., Duan, L., Hudzik, H., Wisła, M.: Basic theory of p-Amemiya norm in Orlicz spaces(1 $\leq$ $p \leq \infty)$ : extreme points and rotundity in Orlicz spaces endowed with these norms. Nonlinear Anal. 69, 1796-1816 (2008)

11. Cui, Y.A., Hudzik, H., Li, J., Wisła, M.: Strongly extreme points in Orlicz spaces equipped with the p-Amemiya norm. Nonlinear Anal. 71, 6343-6364 (2009)

12. Cui, Y., Hudzik, H., Petrot, N.: Basic topological and geometric properties of Cesàro-Orlicz spaces. Proc. Indian Acad. Sci. Math. Sci. 115(4), 461-476 (2005)

13. Cui, Y.A., Hudzik, H., Szymaszkiewicz, L., Wang, T.F.: Criteria for monotonicity properties of MusielakOrlicz spaces equipped with the Amemiya norm. J. Math. Anal. Appl. 303, 376-390 (2005)

14. Cui, Y.A., Hudzik, H., Wisła, M.: Monotouicity properties and dominated best approximation problems in Orlicz spaces equipped with the p-Amemiya norm. J. Math. Anal. Appl. 432, 1095-1105 (2015)

15. Cui, Y.A., Hudzik, H., Li, J., Wisła, M., Wlaźlak, K.: Non-squareness properties of Orlicz spaces equipped with the p-Amemiya norm. Nonlinear Anal. 75, 3973-3993 (2012)

16. Foralewski, P., Hudzik, H., Kaczmarek, R., Krbec, M.: Characteristic of monotonicity of Orlicz function spaces equipped with the Orlicz norm. Comment. Math. 53(2), 421-432 (2013)

17. Foralewski, P., Hudzik, H., Kowalewski, W., Wisła, M.: Monotonicity properties of Banach lattices and their applications-a survey. Ordered Structures and Applications. Springer International Publishing, New York (2016)

18. Foralewski, P., Hudzik, H., Kaczmarek, R., Krbec, M.: Moduli and characteristics of monotonicity in some Banach lattices, Fixed Point Theory Applications, Article ID852346, (2010)

19. Foralewski, P., Hudzik, H., Kaczmarek, R., Krbec, M., Wójtowicz, M.: On the moduli and characteristics of monotonicity of Orlicz-Lorentz function spaces. J. Convex Anal. 20(4), 955-970 (2013)

20. He, X., Cui, Y.A., Hudzik, H.: The fixed point property of Orlicz sequence spaces equipped with the p-Amemiya norm. Fixed Point Theory Appl. 2013, 1-18 (2013)

21. Hudzik, H., Kamińska, A.: Monotonicity properties of Lorentz Spaces. Proc. Am. Math. Soc. 123(9), 2715-2721 (1995)

22. Hudzik, H., Kamińska, A., Mastyło, M.: Monotonicity and rotundity properties in Banach lattices. Rocky Mountain J. Math. 30(3), 933-950 (2000)

23. Hudzik, H., Kaczmarek, R.: Moduli and characteristics of monotonicity in general Banach lattices and in Orlicz spaces in particular. Nonlinear Anal. 70(9), 3407-3423 (2009)

24. Hudzik, H., Kurc, W.: Monotonicity properties of Musielak-Orlicz spaces and dominated best approximation in Banach lattices. J. Approx. Theory 95, 353-368 (1998) 
25. Hudzik, H., Liu, X.B., Wang, T.F.: Points of monotonicity in Musielak-Orlicz function spaces endowed with the Orlicz norm. Publ. Math. Debrecen 60(3-4), 385-403 (2002)

26. Hudzik, H., Liu, X.B., Wang, T.F.: Points of monotonicity in Musielak-Orlicz function spaces endowed with the Luxemburg norm. Arch. Math. 82(6), 534-545 (2004)

27. Hudzik, H., Maligranda, L.: Amemiya norm equals Orlicz norm in general. Indag. Math.(N.S.) 11(4), 573-585 (2000)

28. Hudzik, H., Narloch, A.: Local monotonicity structure of Calderón-Lozanovskii spaces. Indag. Math. 15(2), 245-255 (2004)

29. Hudzik, H., Narloch, A.: Relationships between monotonicity and complex rotundity properties with some consequences. Math. Scand. 96(2), 289-306 (2005)

30. Kantorovich, L.V., Akilov, G.P.: Functional Analysis in Normed Spaces, Pergamon Press, Oxford New York Toronto Sydney Paris Frankfurt (1982) (translated from Russian)

31. Krasnoselskii, M. A., Rutickii, Ya. B.: Convex Functions and Orlicz Spaces, Gröningen, (1961) (translated from Russian)

32. Kurc, W.: Strictly and uniformly monotone Musielak-Orlicz spaces and applications to best approximation. J. Approx. Theory 69(2), 173-187 (1992)

33. Kurc, W.: Strictly and uniformly monotone sequential Musielak-Orlicz spaces. Collect. Math. 50(1), 1-17 (1999)

34. Lindenstrauss, J., Tzafriri, L.: Classical Banach spaces II : Function spaces. Springer, New York (1979)

35. Luxemburg, W.A.J.: Banach function spaces. Thesis, Delft (1955)

36. Maligranda, L.: Orlicz Spaces and Interpolation, Seminars in Mathematics, Universidad Estadual de Campinas, (1989)

37. Medzhitov, A., Sukochev, P.: The property (H) in Orlicz spaces. Bull. Polish Acad. Sci. Math. 40(1), 5-11 (1992)

38. Musielak, J.: Orlicz spaces and modular spaces. Lecture Notes in Mathematics, vol. 1034. Springer, New York (1983)

39. Orlicz, W.: Über eine gewise Klasse von Räumen von Types B, Bull. Int. Pol. Ser. A Kraków, 207-220 (1932)

40. Rao, M.M., Ren, Z.D.: Applications of Orlicz spaces. Marcel Dekker Inc, New York (2002)

41. Wnuk, W.: Banach lattices with order continuous norms. Polish Scientific Publishers PWN, Warszawa (1999)

Publisher's Note Springer Nature remains neutral with regard to jurisdictional claims in published maps and institutional affiliations. 